\section{Skeletal dysplasia with unusual visceral manifestations}

\section{S R Ahuja, S Karande, M V Kulkarni}

\section{Answers on p 107.}

$\Lambda$ boy aged 4.5 years, born of a third degree consanguineous marriage presented with short stature, a lump on the left side of the abdomen that had been seen since he was 7 months of age, and noisy breathing on and off for the past two years. There was no history of jaundice, fever, bleeding from any site, blood transfusion, or fractures. The birth history and milestones were normal. His two siblings were normal.

On examination, the child was grossly stunted. The height was $78 \mathrm{~cm}$, weight 10 $\mathrm{kg}$, and the head circumference $48 \mathrm{~cm}$. The height and weight were markedly below the fifth percentile. The upper segment: lower segment ratio was 1.34:1 indicating proportionate short stature. There was marked pallor but no icterus,

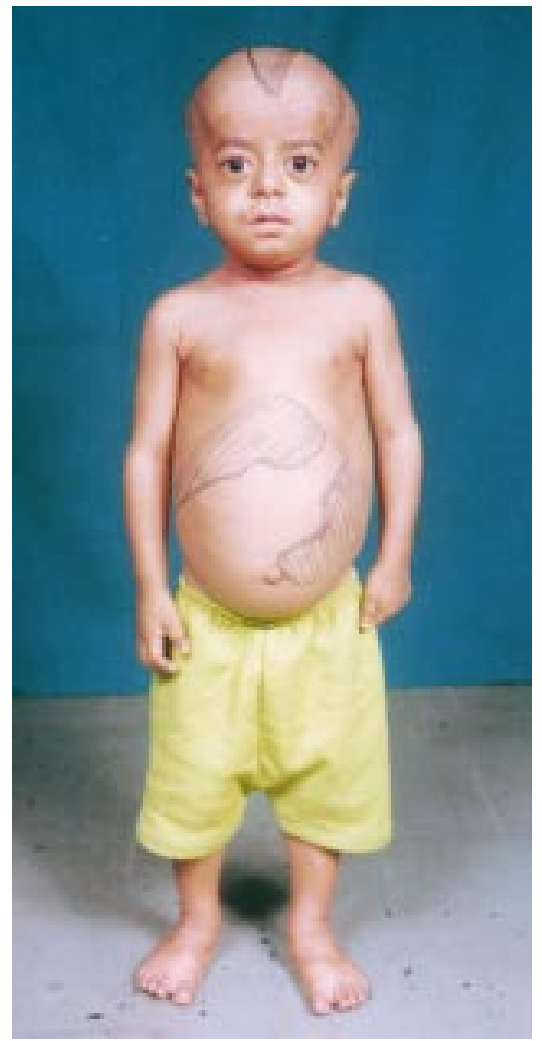

Figure 1 Clinical photograph of child showing widely open anterior fontanelle (delineated by markings), bilateral proptosis, frontal bossing, a narrow chin, and massive splenohepatomegaly (delineated by markings). (Reproduced with permission.) oedema, petechiae, or significant lymphadenopathy. There was marked frontoparietal bossing, the sutures were wide open, the anterior fontanelle measured 12 $\times 12 \mathrm{~cm}$ and communicated with an open

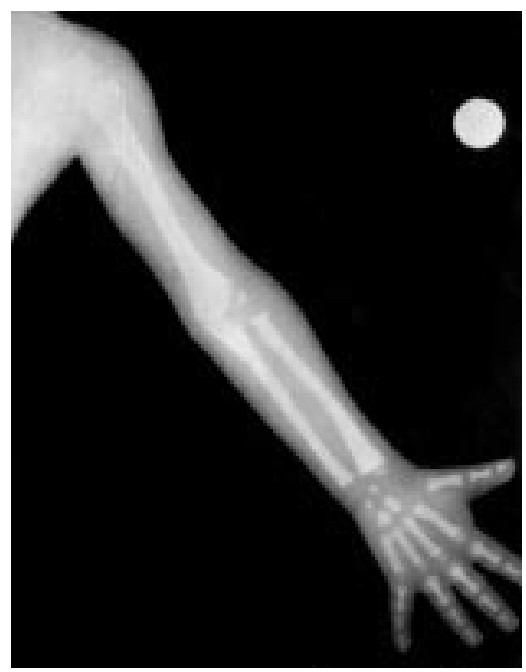

Figure 2 Radiograph of the left upper limb showing generalised hyperdensity of bones. The medullary canals are not visualised. Distal phalanges are hypoplastic.

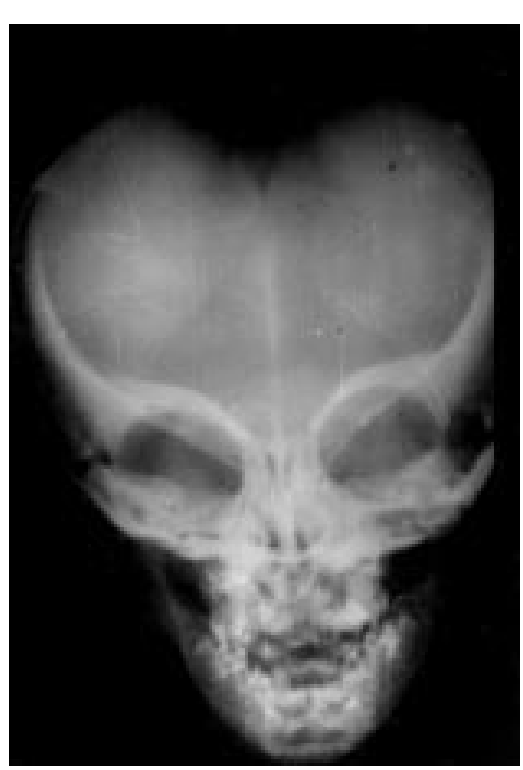

Figure 3 Radiograph of the skull showing a wide open anterior fontanelle and absence of frontal sinuses. posterior fontanelle. There was bilateral proptosis, a small nose, and a small poorly developed mandible (fig 1). The uvula was long and the palate high arched. The teeth showed marked caries and an irregular arrangement. The hands were small with short and stubby fingers. The feet were small with short and stubby toes.

On abdominal examination, the spleen was enlarged $13 \mathrm{~cm}$ below the left costal margin and the liver was palpable $5 \mathrm{~cm}$ below the right costal margin, both were firm and non-tender (fig 1). Fundoscopy revealed bilateral papilloedema with no evidence of secondary optic atrophy. The rest of the systemic examination was normal.

Investigations revealed a haemoglobin concentration of $68 \mathrm{~g} / \mathrm{l}$ and leucocyte count $14.4 \times 10^{9} /$; differential count was neutrophils $46 \%$, lymphocytes $50 \%$, and monocytes $4 \%$. The platelet count was 77 $\times 10^{9} / 1$ and corrected reticulocyte count was $0.9 \%$. The peripheral smear showed normocytic and normochromic red blood cells and a few target cells. There were no abnormal cells or parasites seen. Osmotic fragility and haemoglobin electrophoresis were normal. Sickling test and Coombs test were negative. Serum calcium concentration was $2.3 \mathrm{mmol} / \mathrm{l}$, serum phosphorus $1.54 \mathrm{mmol} / \mathrm{l}$, and alkaline phosphatase was low: $139 \mathrm{U} / \mathrm{l}$ (normal range 210-810 U/1). The child's IQ was 95.

Skeletal survey showed a generalised and uniform increase in bone density (osteosclerosis) with marked obliteration of the medullary canals (fig 2). Radiography of the skull showed open anterior and posterior fontanelles, separated sutures, wormian bones, non-pneumatised frontal sinuses, and a sclerotic skull base (fig 3). The mandibular angle was obtuse.

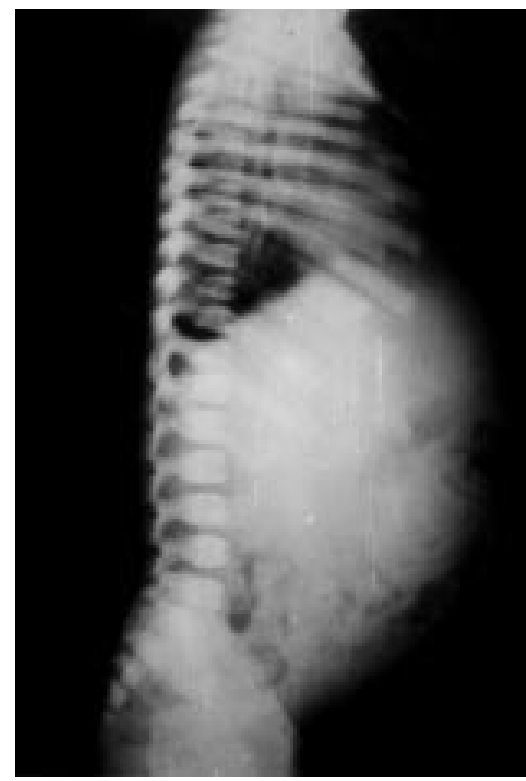

Figure 4 Radiograph of the chest and abdomen lateral view showing broad, thick, and dense ribs. 
Radiography of the chest showed thick and dense ribs (fig 4) The clavicles were normal. Radiography of the hands showed hypoplasia and narrowing of distal phalanges (fig 2). There was no evidence of fractures.

Computed tomography of the brain was normal. Audiometry revealed normal hearing in both ears. Bone marrow aspiration showed a leucoerythroblastic reaction indicative of bone marrow inva- sion (myelophthisis). The myeloid:erythroid ratio was $2: 1$ (normal).

\section{QUESTIONS}

(1) What is the diagnosis?

(2) What is the most important differential diagnosis?

(3) What complications can be anticipated in this case?

Postgrad Med J 2002;78:99-100

\section{Authors' affiliations}

S R Ahuja, S Karande, M V Kulkarni, Development Disorders Clinic, Department of Pediatrics, LTM Medical College and LTMG Hospital, Sion, Bombay 400 022, India

Correspondence to: Dr Karande; karandesunil@yahoo.com

Submitted 20 April 2001

Accepted 3 July 2001

\section{Complications associated with influenza infection}

\section{S D West, N J Brunskill}

\section{Answers on p 107.}

$\Lambda^{\mathrm{n}}$ otherwise healthy non-smoking 59 year old welder gave a two week history of flu-like symptoms and a one week history of progressive shortness of breath, pleuritic chest pain, and cough productive of purulent sputum.

On examination he was unwell with a pyrexia and an oxygen saturation of $87 \%$ on air. He had atrial fibrillation with a pulse of 150 beats/min and blood pressure of 109/69 mm Hg. He was jaundiced with epigastric and right upper quadrant abdominal tenderness. Respiratory examination revealed tachypnoea, right mid-zone coarse crepitations, and a right sided pleural rub.

Investigations showed a raised white cell count with a neutrophilia, significant renal impairment with a urea concentration of $23.3 \mathrm{mmol} / \mathrm{l}$ and creatinine of 248 $\mu \mathrm{mol} / \mathrm{l}$, oxygen pressure of $7.1 \mathrm{kPa}$ and carbon dioxide pressure of $2.5 \mathrm{kPa}$ on air, and an abnormal chest radiograph with consolidation of his right middle lobe (fig 1A). Liver function tests were also abnormal with a bilirubin of $137 \mu \mathrm{mol} / \mathrm{l}$ and alanine aminotransferase of 123 U/1. His blood cultures grew Streptococcus pneumoniae and his influenza A titre was markedly raised.

Despite starting intravenous amoxicillin and clarithromycin, he developed bronchial breathing in his left lower lung. His renal function worsened and he became anuric. Ultrasound scan showed normal sized kidneys with no hydronephrosis. The gall bladder was distended but the liver appeared normal. $\mathrm{He}$ was started on haemodialysis and showed initial clinical improvement.

Six days after admission he deteriorated. He developed a spiking pyrexia and expectorated copious amounts of purulent sputum. A leucocytosis developed. A further chest radiograph was taken which is shown here (fig 1B). Sputum culture grew Pseudomonas aeruginosa. His antibiotic therapy was subsequently changed to imipenem and metronidazole.

\section{QUESTIONS}

(1) What do the chest radiograph (fig 1B) and computed tomogram of the thorax (fig IC) show and how would the clinical and radiological findings be best treated?

(2) Is the high influenza A titre relevant to this case?

(3) What is the cause of his acute renal failure?

(4) What is the cause of his abnormal liver function tests?

Postgrad Med J 2002;78: 100
Authors' affiliations

S D West, N J Brunskill, Department of Nephrology, Leicester General Hospital, Gwendolen Road, Leicester LE5 4PW, ÚK

Correspondence to: Dr Brunskill

Submitted 16 March 2001

Accepted 14 June 2001
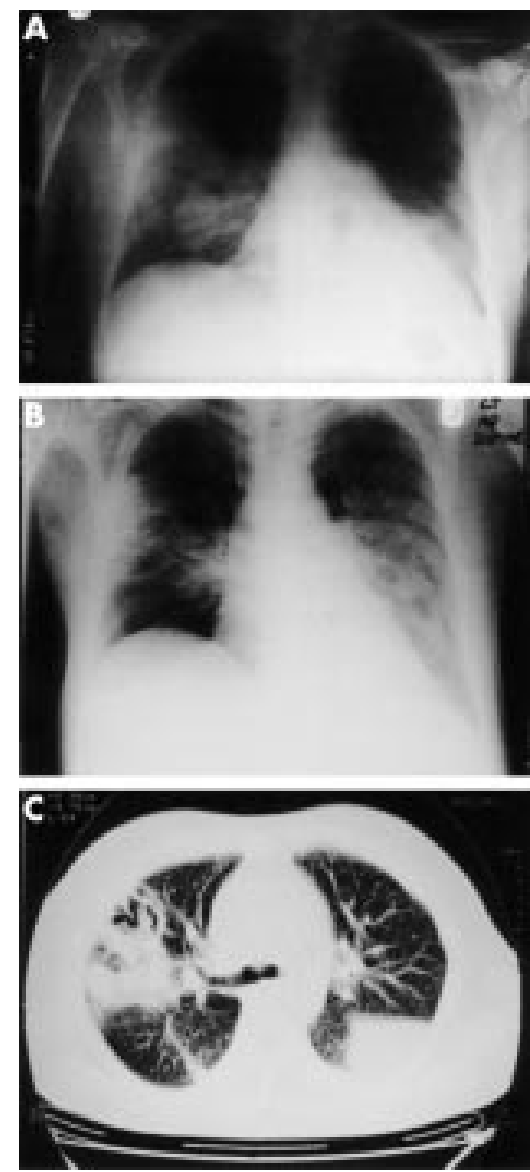

Figure 1 Radiological investigations: (A) chest radiography on admission; (B) chest radiography six days after admission; (C) computed tomography of the chest six days after admission. 